\title{
Fast Face Recognition Based on 2D Fractional Fourier Transform
}

\author{
Hao Luo ${ }^{12}$ \\ Department of Information Science and Engineering, Wuhan University of Science and Technology, \\ Wuhan, 430081, China \\ E-mail: $764724606 @$ qq. com

\section{Yu Wei ${ }^{3}$} \\ Department of Information Science and Engineering, Wuhan University of Science and Technology, \\ Wuhan, 430081, China \\ E-mail: $872463236 @$ qq. com
}

\section{Man Zhou}

Department of Information Science and Engineering, Wuhan University of Science and Technology, Wuhan, 430081, China

E-mail: 2903256411 @qq. com

In order to overcome the deficiency of computational complexity and low accuracy of traditional face recognition algorithm under complex surroundings, this paper proposes a novel face recognition method based on 2D-FrFT. Firstly, we construct Gaussian skin color model to segment potential face region; then adaptive template matching and secondary matching algorithm are adopted to eliminate the impact of background and reduce computational cost in the process of matching.Meanwhile, LBP operator encodes the amplitude and phase information of 2D-FrFT as texture feature. Eventually, a nearest neighbor classifier is employed for classification. Our proposed approach is examined on the public available database. The experimental results demonstrate that this method is not only simple and robust, but efficient in recognition speed and rate.

CENet2017

22-23 July 2017

Shanghai, China

\footnotetext{
${ }^{1}$ Speaker

${ }^{2}$ Corresponding Author

${ }^{3}$ This work is supported by "the National Natural Science Foundation of China" (Project No.: 61673304).
} 


\section{Introduction}

Face recognition(FR) has received a significant interest in the field of pattern recognition, image analysis and artificial intelligence. FR technique is still a challenging task due to the fact that face images are vulnerable to some variation factors, such as, illumination, expressions, occlusion, etc[1]. Face detection is the first primary step and skin color is regarded as an important feature information. Many face detection algorithms based on skin color have been proposed[2-3]. In the literature of face recognition, the main approaches applied for face feature extraction are often classified into two major categories: namely, global feature analysis and local feature description. Global feature method is usually utilized for coarse representation. However, a large and representative training sets are required for learning. On the contrary, local feature description can overcome the above shortcomings and aim to extract more local part features. LBP feature is one of the most powerful texture description proposed by Ojala [4] and has been widely applied in texture classification and face image analysis.

As a generalized form of the conventional Fourier transform, FrFT[5] is considered as a new tool for time-frequency analysis. It executes a rotation to any angle in FrFT domain and facial image data is also changeable signal, thus, it is a better way to apply FrFT to image analysis. To the end, this paper proposes an approach for fast face recognition based on 2DFrFT. We utilize color information to segment face region; then two times template matching are introduced, afterwards, LBP operator encodes the fused feature of FrFT as texture feature; finally, a nearest neighbor classifier is employed for classification.

The rest of this paper is organized as follows: Section 2 demonstrates the establishment of Gaussian model and face segmentation. Section 3 mainly introduces the implementation of two times template matching. Section 4 illustrates 2DPCA and classification measure. In Section 5, the experimental results are present and afterwards including remarks are made in Section 6.

\section{Skin Color Segmentation}

\subsection{Light Compensation}

The given images are usually inevitably affected owing to the change of light, which will lead to deviate from real skin color. Therefore, input image should be performed light compensation before face segmentation. In this paper, we adopt a pre-processing light compensation technique called "Gray World Theory"[6] to conduct color correction. The processing result is shown in Figure 1.

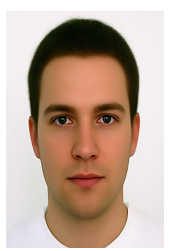

(a)

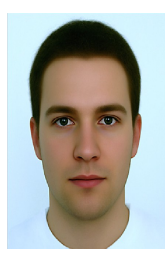

(b)

Figure 1: Light Compensation Processing (a) color image containing face; (b) the result of color balance. 


\subsection{Face Detection}

Compared with other color spaces, $\mathrm{YCbCr}$ provides an excellent space for luminance and chrominance separability and exhibits better clustering property in distinguishing skin color from background which is more suitable for face detection [7]. In the following, Gaussian model is established to segment face candidate region, then morphological operation is used for noise filtering, afterwards, according to the geometric relationships of human face (ie. face aspect ratios), eventually the coarse location of facial area is shown in Figure 2.

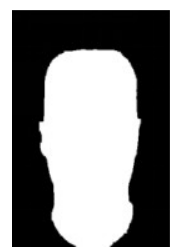

(a)

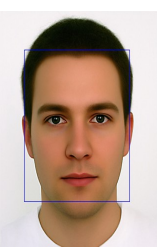

(b)

Figure 2: Coarse Localization of Face: (a) morphological operations; (b) face detection result.

\section{Fast Face Recognition Based on FrFT}

\subsection{D Fractional Fourier Transformation}

An extension of Fourier transform, FrFT has become a potential tool for time-varying and non-stationary signal processing. It can be interpreted as a rotation of signals in time-frequency plane [8]. The kernel of 2D-FrFT is defined as follows:

$$
\begin{aligned}
& K p_{1}, p_{2}(s, t, u, v)=\frac{\sqrt{1-j \cot \alpha} \sqrt{1-j \cot \beta}}{2 \pi} . \\
& \exp \left[\frac{j\left(s^{2}+u^{2}\right)}{2 \tan \alpha}-\frac{j s u}{\sin \alpha}\right] \exp \left[\frac{j\left(t^{2}+v^{2}\right)}{2 \tan \beta}-\frac{j t v}{\sin \beta}\right]
\end{aligned}
$$

Where $p_{1}$ and $p_{2}$ are different orders of FrFT, $\alpha=p_{1} \pi / 2$ and $\beta=p_{2} \pi / 2$ represent the rotation angel after transformation.

Mathematically, assuming transform $p_{1}$ and $p_{2}$ in definite, 2D-FrFT image signal $f(x, y)$ combines the transform kernel of 2D-FrFT can be expressed as:

$$
F p_{1}, p_{2}=\int_{-\infty}^{+\infty} \int_{-\infty}^{+\infty} f(s, t) K p_{1}, p_{2}(s, t, u, v) d s d t
$$

In fact, 2D-FrFT kernel function is separable in two-dimensional space and it is equivalent to employ FrFT on the two different parameters of the signal

$$
K p_{1}, p_{2}(s, t, u, v)=K p_{1}(s, u) \cdot K p_{2}(t, v)
$$

\subsection{Amplitude and Phase Information of Image in FrFT Domain}

2D-FrFT is a complex transformation which includes both amplitude information and phase information after fractional transformation. Suppose $f(x, y)$ is gray image information, $F(\mu, v)$ represents the FrFT transformation, $F r T_{2 \mathrm{D}}$ is $2 \mathrm{D}-\mathrm{FrFT}$ operator. Based on the above description, we have

$$
F(\mu, v)=F r T_{2 \mathrm{D}}[f(x, y)]=A(\mu, v) \cdot P(\mu, v)
$$

Where $A(\mu, v)=|F(\mu, v)|$ refers to the amplitude information after feature extraction, 
$P(\mu, v)=F(\mu, v) / A(\mu, v)$ denotes the phrase information.

The 2D-FrFT possesses the dual properties of periodicity and symmetry in the FrFT domain. Without loss of generality, we select two same values orders $p_{1}=p_{2}$ in the following experiment. Figure 3 corresponds to the amplitude and phase representation features of image in the order interval $(0.1,1.0)$ and step sets 0.1 , that is, image transformation from time domain to frequency domain. Fig. (a) shows that images energy clustering characteristics in fractional domain are gradually rising with the transform order increasing, when $p=1$, the FrFT is analogous to the normal Fourier transform, at the same time, the energy distribution rate reaches the maximum. Fig. (b) displays that contour texture features of human face slowly decreass when the order continually changes from 0.1 to 1.0 , in addition, the results also indicate phase feature apparently highlight more edges and lines information, especially some detail features. Based on the above analysis, amplitude and phase information better depict the performance of facial features and reflect obvious face information. Hence, in the work, we employ their complementary information by means of fusing the magnitude and phase information of the FrFT for face recognition.

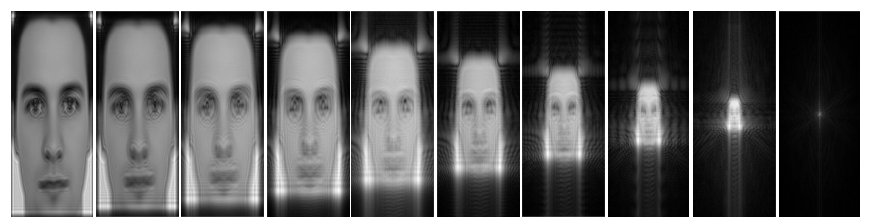

(a)

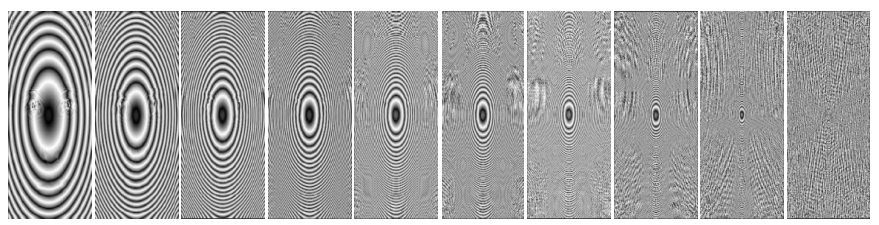

(b)

Figure 3: (a) Amplitude information of the original image in fractional domain; (b) Phase information of the input image in fractional domain.

\subsection{Local Binary Pattern}

LBP operator has been proved as a powerful discriminative descriptor for texture classification. We can obtain final binary result by computing each neighborhood pixel $f_{p}$ with its center pixel value $f_{c}$. If all neighbors have higher or equal value of central pixels, ' 1 ' is assigned; otherwise, ' 0 ' is assigned. Formally, LBP operator is described based on eq.(3.5). Fig. 4 gives an example of the basic LBP encoding process.

$$
\begin{aligned}
& S\left(f_{p}-f_{c}\right)=\left\{\begin{array}{ll}
1 & f_{p} \geqslant f_{c} \\
0 & f_{p}<f_{c}
\end{array} \quad L B P=\sum_{p=0}^{7} S\left(f_{p}-f_{c}\right) 2^{p}\right. \\
& \rightarrow \rightarrow \begin{array}{|c|c|c|}
\hline 56 & 20 & 34 \\
\hline 128 & 22 & 12 \\
\hline 6 & 18 & 78 \\
\hline
\end{array} \rightarrow \begin{array}{|c|c|c|c|}
\hline 1 & 0 & 1 \\
\hline 1 & & 0 \\
\hline 0 & 0 & 1 \\
\hline
\end{array} \\
& L B P_{f_{c}}=149 \longleftarrow 2^{0}+2^{2}+2^{4}+2^{7}
\end{aligned}
$$

Figure 4: Bsic LBP Operator 


\subsection{Adaptive Matching of Local Feature}

Skin and facial feature are regarded as the most important features in face image, in order to achieve better recognition results, two times template matching methods based on the combination of local and global feature are proposed. The average face template is derived from manual cropping face region and selected from standard image samples, and conducted to the processing of histogram equalization to calculatethe average of faces. Then we can obtain $24 \times 20$ pixel average face template. In view of the significance of facial feature like eyes, mouth and nose, they will combine average face template as a whole to form template pairs. This paper chooses the templates of eyes, mouth and nose which separately correspond to $20 \times 6$ pixel, $10 \times 7$ pixel and $8 \times 8$ pixel. Figure 5 displays facial feature template. According to the prior knowledge, when searched and compared in candidate region, we firstly utilize facial feature template to enlarge scope nearby, then the average face templates with different aspect ratios are employed later to match faces. The size and deflection angle of facial template can be adjusted based on the possible facial region's size and angle. For each window under detection, if the interrelated coefficient between two image matrix exceeds the threshold value $\theta=0.6$, it will be possible for face region. Then the detection windows will come into the next detection. Subsequently, the amplitude and phase information of different transform orders in FRFT domain are extracted as facial features and LBP operator encodes its texture feature. This method is simple and has better adaptability to different facial size and large deflection angle, but requests a lot of time.
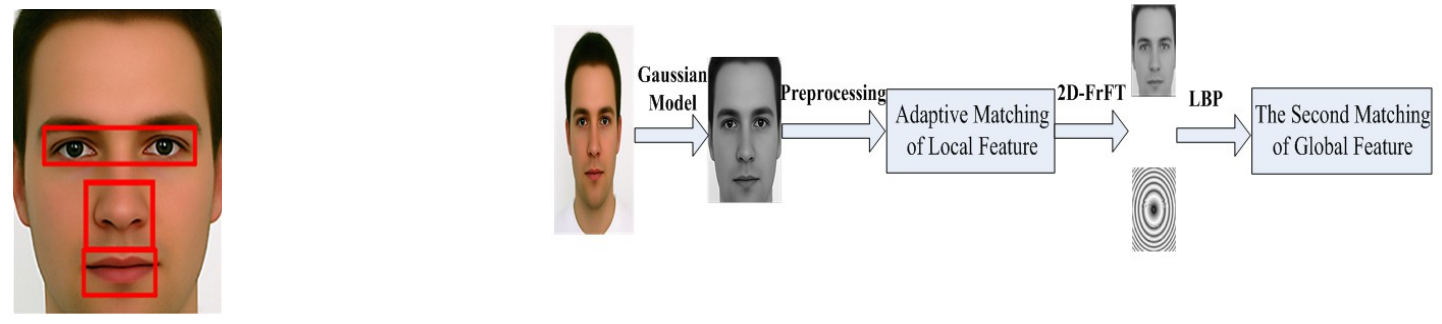

Figure 5: Partial Templates of Face. $\quad$ Figure 6: Framework of Face Recognition.

\subsection{The Second Matching of Global Feature}

After having implemented the initial matching of face template, we can determine whether it contains face region. In order to reduce the amount of data access and improve the matching speed, secondary matching error algorithm is adopted in this paper. The first template matching is rough matching, that is, interval scanning is performed in the detected face region, however, there still exists some similar regions or non-face areas in the detection windows. The second matching is the exact matching, and the entire face with the identified facial feature templates in face block are carried out to scan and screenin the neighborhood of the minimum error point $\left(i_{\text {min }}, j_{\text {min }}\right)$ after the first template matching. In other words, in the diagonal point $\left(i_{\text {min }}-1, j_{\text {min }}-1\right)$ and $\left(i_{\text {min }}-1, j_{\text {min }}+1\right)$ within the rectangle, it is conducted mathematical calculation by Equation (3.6) and obtains the final matching result. If $E(i, j)>E_{0}$, it will suspend current operation to this point and start the next point operation, greatly reduce computation costs in the process of matching. In the following, in the same way, LBP operator 
extracts the fused information of FrFT as texture feature. The overall flow diagram of face recognition is illustrated in Figure 6.

$$
\begin{gathered}
E(i, j)=\sum_{k=1}^{m} \sum_{l=1}^{n}\left|S_{i j}(k, l)-T(k, l)\right| \\
E_{0}=\frac{e_{0} \times(m-1) \times(n-1)}{4}
\end{gathered}
$$

Where $E(i, j)$ represents the matching target, $S_{i j}$ gives the face image, $T(k, l)$ is the matching template, $E_{0}$ refers to the error threshold value, $e_{0}$ is the maximum average error of each points and it is usually set 40 50.

\section{2DPCA Algorithm and Classification Measure}

The traditional PCA must be transformed from 2D image matrices into 1D vector previously, while 2DPCA is put forward by Yany et al[9] which can directly calculate the covariance matrix and less time is required for training.

Suppose $\mathrm{X}$ represents $\mathrm{m}$-dimensional unitary column vector, the basic idea of 2DPCA is that an $\mathrm{m} \times \mathrm{n}$ image matrix $\mathrm{A}$ is projected to $\mathrm{X}$ vector by linear transformation function: $Y=A^{T} X$. In view of of the fact that face image is classified into face area and non- face area via face detection, and given pattern class number is $\mathrm{c}, \mathrm{w}(\mathrm{i})(\mathrm{i}=1, \ldots, \mathrm{c})$ separately express face and non-face class, where n(i) denotes training samples to each class. $\mathrm{A}(1), \mathrm{A}(2), \ldots, \mathrm{A}(\mathrm{N})\left(N=\sum_{i=1}^{2} n_{i}\right)$ refer to all training samples, every sample A(i) $(\mathrm{i}=1, \ldots . \mathrm{N})$ denotes $\mathrm{m} \times \mathrm{n}$ matrix. The total sample covariance matrix is written by the following equation

$$
G_{t}=\frac{1}{N} \sum_{i=1}^{N}\left(A_{i}-\bar{A}\right)\left(A_{i}-\bar{A}\right)^{T}
$$

Where $\bar{A}$ gives the mean matrix of whole training samples. From Eq. (4.1), we can get a non-negative definite matrix $G_{t}$. In fact, $x_{1}, x_{2}, \cdots, x_{d}$ can be obtained by maximizing image scatter criterion

$$
J(X)=X^{T} G_{t} X
$$

Where $\mathrm{X}$ corresponds to the largest eigenvalue of orthonormal eigenvectors. Eventually, 2DPCA optimal project vector is $X_{\text {opt }}=\left[x_{1}, x_{2}, \cdots, x_{d}\right]$.

For each training face samples, feature matrix is calculated by $Y_{k}=A^{T} X_{k}$. As is depicted above, the eigenmatrix of A matrix is $B_{t}=\left[Y_{1}, Y_{2}, \cdots, Y_{d}\right]$. Then, a nearest neighbor classifier based on matrix distance is employed for classification.

$$
d\left(B_{t}, B_{i}\right)=\min \sum_{k=1}^{d}\left\|b_{t k}-b_{i k}\right\|
$$




\section{Experiment Analysis}

\subsection{Sample Database}

In order to verify the effectiveness of our proposed scheme, face images database taken from the California institute of technology are adopted in the experiment. The database contains 27 people and a total of 450 images, and each image size is $869 \times 592$. These face images are respectively selected from different illumination conditions, facial expression and background surroundings. Part of face images is shown in Fig. 7.

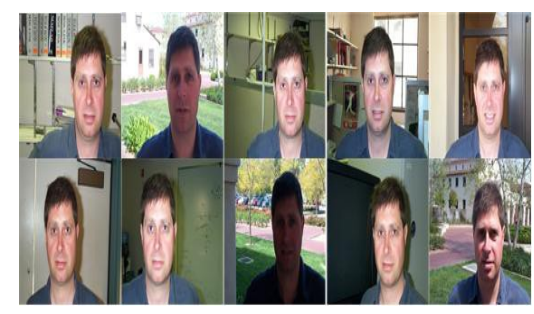

Figure 7: Part of the Face Images

\subsection{Experimental Results}

In the experiment, all face images from our selected database before performing the experiment need to be normalized, 4 images per for person and 108 images in total are chosen randomly as training samples, and the remaining are used to form the testing set. Every experiment repeats 10 times, and takes the average of its experiment as final results. Figure 8 shows the recognition results of amplitude and phase feature information under different transform orders $\mathrm{p}$ with step is set 0.1 .

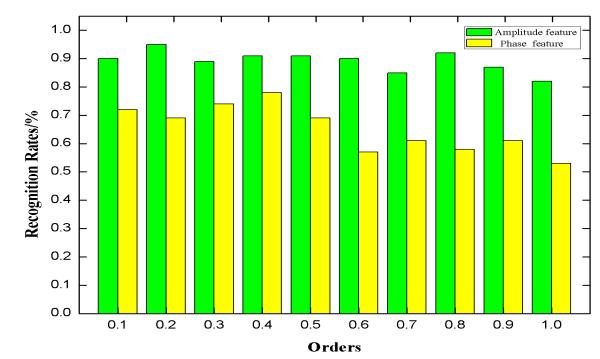

Figure 8: Recognition Rates of Amplitude and Phase Features under Different Transform Orders

As we can clearly observe that Fig. 8 displays a series of different recognition results which indicate that the ability of every order to extract information is different in 2D-FrFT domain. When $\mathrm{p}=1$, that is, the normal Fourier transform, it has the minimum recognition rate. Obviously, with the other different $\mathrm{p}$ values, 2D-FrFT corresponds to different time-frequency information, and reflects different features of the original pattern and has higher recognition rates. Because the orders embody the distribution of energy and information, among them, order 0.2 and 0.4 respectively reach the best recognition rate. In addition, the performance of amplitude feature in a whole surpasses phase feature, on account that phase information is instabley andchangeable, while amplitude information mainly relies on the spatial distribution of image gray value and is relatively steady. 


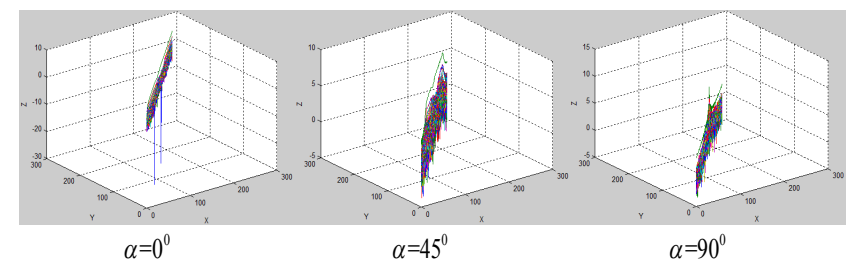

(a)

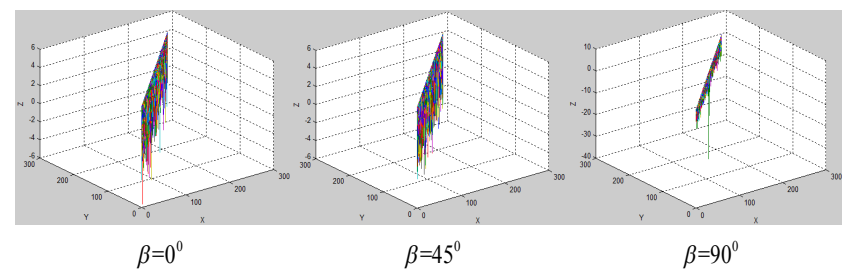

(b)

Figure 9: (a) Amplitude information of three-dimension maps corresponds to different rotation angles; (b) Phase information of three-dimension maps corresponds to different rotation angles.

In this section, we demonstrate the corresponding relationship between the amplitude and phase feature information and different rotation angles from the perspective of 3D maps, which can be seen from Figure 9. With our mathematical analysis illustrated in the previous part, the rotation angle changes from $0^{0}$ to $90^{0}$ corresponding to the transform order turns from 0 to 1 , it is evident that $3 \mathrm{D}$ maps of different rotation angles greatly varies and when rotation angle increases from $0^{0}$ to $45^{\circ}$, the magnitude feature is dominated, while turns from $45^{0}$ to $90^{\circ}$, phase information is more significant. Hence, it is meaningful to utilize their complementary information to extract discriminative feature for face recognition.

In order to further evaluate the performance of proposed scheme, in the following work, we will compare our method with two classical algorithms as shown in table 1 . All methods are conducted on the same image set. Table 1 lists the results of three approaches on recognition rate and the running time costs.

\begin{tabular}{|c|c|c|}
\hline Method & Recognition rate $/ \%$ & Time $/ \mathrm{ms}$ \\
\hline The algorithm based skin color[10] & 82.14 & 137 \\
\hline 2DPCA & 87.57 & 370 \\
\hline The proposed method & 95.24 & 204 \\
\hline
\end{tabular}

Table 1: Recognition Results of Different Algorithms.

The results are shown in Table 1. In terms of the recognition rate, obviously our proposed algorithm outperforms the methods based on skin color facial feature and 2DPCA, and it yields the highest recognition rate of 95.24 percent because the facial feature approach and 2DPCA algorithm will be misjudged the non-skin color region to be face area and part of face information will be left out. On the contrary, our proposed method not only adopts two times template matching to effectively overcome the impact of backgrounds, but takes full advantage of the complementary information of 2D-FrFT to extract its feature, which enhances discriminative feature information of images and removes redundancy. As to time consumption, 
skin color facial feature algorithm requires less time than the other methods. Therefore, the validity of our proposed method is confirmed by the experiment.

\section{Conclusion}

This paper presents a new face recognition method by means of fusing skin color information, matching template and 2D-FrFT. Adaptive template matching is regarded as a rough classifier and second matching algorithm is introduced which can effectively overcome the impact of background and improve the matching speed. What's more, we take full advantage of their complementary information of 2D-FrFT to extract significant discriminative feature and illustrate it from the perspective of 3D maps. The experimental results on public database demonstrate that our proposed scheme can well extract facial features and achieve higher recognition rate compared with other methods, while for the variations of facial pose, expression and occlusion are robuster.

\section{References}

[1] Z. P. Hu, J. Li. Face recognition of joint sparse representation based on low-rank subspace recovery [J]. Acta Electronica Sinica. 5: 024(2013). (In Chinese)

[2] L. Nanni, A. Lumini, F. Dominio, et al. Effective and precise face detection based on color and depth data[J]. Applied Computing and Informatics. 10(1): 1-13(2014).

[3] D. Ghimire, J. Lee. A robust face detection method based on skin color and edges[J]. Journal of Information Processing Systems. 9(1): 141-156(2013).

[4] T. Ojala, M. Pietikainen, T. Maenpaa. Multiresolution gray-scale and rotation invariant texture classification with local binary patterns[J]. IEEE Transactions on Pattern Analysis and Machine intelligence. 24(7): 971-987(2002).

[5] R. Tao, L. Qi, Y. Wang. Theory and applications of the fractional Fourier transform[J]. Beijing: Publisher of Tsinghua University. 2004. (In Chinese)

[6] KHB. Ghazali, J. Ma, R. Xiao. An innovative face detection based on $\mathrm{YCgCr}$ color space [J]. Physics Procedia. 25: 2116-2124(2012).

[7] T. H, Huang, Y. M. Yu, X. G. Qi. A High-performance Skin Segmentation Method[J]. Procedia Engineering. 15: 608-612(2011). (In Chinese)

[8] J. Yang, J. Y. Yhang, D. Zhang et al. Feature fusion: parallel strategy vs. serial strategy[J]. Pattern Recognition. 36(6): 1369-1381(2003). (In Chinese)

[9] J. Yang, D. Zhang, A. F. Frangi, et al. Two-dimensional PCA: a new approach to appearance-based face representation and recognition[J]. IEEE Transactions on Pattern Analysis and Machine Intelligence. 26(1): 131-137(2004). (In Chinese)

[10] X. Wang, H. X. Zhao. On fast face detection based on improved complexion space and geometry features[J]. Computer Applications and Software. 32(2): 151-154 (2015). (In Chinese) 